\title{
1 FADU: A Feature Counting Tool for Prokaryotic RNA-Seq \\ 2 Analysis
}

3 Matthew Chung ${ }^{1,2}$, Ricky S. Adkins ${ }^{1}$, Amol C. Shetty ${ }^{1}$, Lisa Sadzewicz ${ }^{1}$, Luke J. Tallon ${ }^{1}$ Claire M. Fraser ${ }^{1,3}$,

4 David A. Rasko ${ }^{1,2}$, Anup Mahurkar ${ }^{1}$, and Julie C. Dunning Hotopp ${ }^{1,2,4, *}$

$5 \quad{ }^{1}$ Institute for Genome Sciences, University of Maryland School of Medicine, Baltimore, MD 21201, USA

$6{ }^{2}$ Department of Microbiology and Immunology, University of Maryland School of Medicine, Baltimore,

7 MD 21201, USA.

$8{ }^{3}$ Department of Medicine, University of Maryland School of Medicine, Baltimore, MD 21201, USA.

$9{ }^{4}$ Greenebaum Cancer Center, University of Maryland School of Medicine, Baltimore, MD 21201, USA.

11 MC: mattchung@umaryland.edu

12 RSA: sadkins@som.umaryland.edu

13 ACS: ashetty@som.umaryland.edu

14 LS: Isadzewicz@som.umaryland.edu

15 UT: litallon@som.umaryland.edu

16 CMF: $\underline{\text { cmfraser@som.umaryland.edu }}$

17 DAR: drasko@som.umaryland.edu

18 AM: amahurkar@som.umaryland.edu

19 JCDH: jdhotopp@som.umaryland.edu 


\section{Abstract}

21 Motivation: The major algorithms for quantifying transcriptomics data for differential gene expression

22 analysis were designed for analyzing data from human or human-like genomes, specifically those with

23 single gene transcripts and distinct transcriptional boundaries that extend beyond the coding sequence

24 (CDS) as identified through expressed sequence tags (ESTs) or EST-like sequence data. Some eukaryotic

25 genomes and all, or nearly all, bacterial genomes require alternate methods of quantification since they

26 lack annotation of transcriptional boundaries with EST or EST-like data, have overlapping transcriptional

27 boundaries, and/or have polycistronic transcripts.

28 Results: An algorithm was developed and tested that better quantifies transcriptomics data for

29 differential gene expression analysis in organisms with overlapping transcriptional units and

30 polycistronic transcripts. Using data from standard libraries originating from Escherichia coli and

31 Ehrlichia chaffeensis, and strand-specific libraries from the Wolbachia endosymbiont wBm, FADU can

32 derive counts for genes that are missed by HTSeq and featureCounts. Using the default parameters with

33 the E. coli data, FADU can detect transcription of 51 more genes than HTSeq in union mode and 21

34 genes more than featureCounts, with 42 and 18 of these features being $\leq 300 \mathrm{bp}$, respectively. Due to its

35 ability to derive counts for otherwise unrepresented genes without overstating their abundance, we

36 believe FADU to be an improved tool for quantifying transcripts in prokaryotic systems for RNA-Seq

37 analyses.

39 implemented using Python3 and requires the PySAM module (version 0.12.0.1 or later).

Contact: jdhotopp@som.umaryland.edu 


\section{$41 \quad 1$ Introduction}

42 A typical analysis pipeline for a gene expression analysis of transcriptomics sequencing data involves: (a)

43 mapping sequencing reads to a whole genome transcriptome assembly with an aligner like Bowtie

44 (Langmead, et al., 2009), BWA (Li and Durbin, 2009), HISAT (Kim, et al., 2015), or STAR (Dobin, et al.,

45 2013); (b) counting reads or fragments for each gene with a tool like HTSeq (Anders, et al., 2015) or

46 Subread featureCounts (Liao, et al., 2014); and (c) finding differentially expressed genes through the use

47 of tools like DESeq (Anders and Huber, 2010) and edgeR (Robinson, et al., 2010). Most of these tools

48 were designed to analyze human data, and as such, they carefully consider important issues that affect

49 these analyses, such as transcript splicing. However, important and relevant genomic features in other

50 organisms complicate transcriptomics analyses in ways unaddressed with this human-centric focus, for

51 example the polycistronic transcripts of bacterial operons.

52 Most commonly identified in prokaryotes, operons are transcriptional units that encode polycistronic

53 transcripts with multiple coding sequences (CDSs). This allows for the coordinated transcription and

54 regulation of all the genes in an operon. As an example, the lac operon encodes a permease for

55 transporting lactose into the cell and a $\beta$-galactosidase which converts lactose to galactose and glucose,

56 allowing for the cis-regulation of multiple functional related genes under a single promoter (Lewis,

57 2013).

58 Presently, the two most popular tools for transcriptome analyses are HTSeq (Anders, et al., 2015) and

59 Subread featureCounts (Liao, et al., 2014). Although in most cases, both tools have no issue quantifying

60 transcripts for specific genes, issues arise when a single fragment can be assigned to multiple genes. By

61 default, both HTSeq and featureCounts bin these reads as ambiguous, rather than assigning them to a

62 specific gene. While this may not be as significant of a problem in eukaryotic systems, the features of a

63 prokaryotic genome, namely the smaller gene size, smaller genome size, and the presence of operons, 
make it difficult for HTSeq and featureCounts to quantify smaller genes, especially those within operons that are smaller than the library insert size.

Here, we test how operons and polycistronic transcripts confound HTSeq and featureCounts, leading to a lack of sequencing data for small genes within operons. We developed a new tool, Feature Aggregate Depth Utility (FADU) to quantify transcription in bacterial genomes. We test FADU on multiple bacterial genomes to demonstrate its utility at capturing sequence data for these underrepresented genes.

\section{System and Methods}

\subsection{Availability of data sets}

Three data sets were used in all analyses consisting of RNA-Seq paired-end data from standard, nonstranded libraries originating from (a) E. coli and (b) E. chaffeensis and stranded libraries from (b) wBm. The sequencing reads for the three datasets can be found in the NCBI Sequencing Read Archive at the following accession numbers, respectively: (pending), SRX485438, and SRX2505171.

\subsection{FADU, featureCounts, and HTSeq comparisons}

For comparative analyses, FADU was run using -count_by fragment and all other default options. HTSeq v0.10.0 (Anders, et al., 2015) was run using default settings while changing the mode for mode-specific analyses. Subread featureCounts v1.6.1 (Liao, et al., 2014) was run using the -p option to specify counting by fragments and/or - $\mathrm{O}$ or -fractional to specify counting different methods of counting ambiguous reads depending on the analysis. Unrooted dendrograms were generated using the $R$ package APE v5.0 (Analysis of Phylogenetics and Evolution) (Paradis, et al., 2004; Popescu, et al., 2012). Bootstrap values were obtained using the R package pvclust v2.0-0 (Suzuki and Shimodaira, 2006). The principal component analysis was performed using the R packages FactoMineR v1.39 (Le, et al., 2008) and factoextra v1.0.5 (http://sthda.com/english/rpkgs/factoextra/).

\section{Algorithm}




\section{3.1 Creating a mapping index using an annotation file}

88 A mapping index is created that contains each position in the reference genome. For each feature

present in the GFF3 or GTF annotation input file, coordinates are marked in the mapping index for each of the features' positions. If the reads are 'stranded' or 'reverse-stranded', a separate mapping index is created and marked for each strand. Each of these coordinates are marked using the features' attribute id. At positions shared by multiple features, the position will be marked as an overlap between two features. These positions, along with positions absent of any feature, will be excluded from downstream feature count calculations. From this, a statistics file will be written that contains the following information for each feature: (a) strand, (b) length of feature, (c) number of coordinates mapping solely to that gene, (d) proportion of non-overlapping coordinates compared to length of feature.

\section{3.2 Calculating read/fragment counts for each feature}

98 For each BAM file, the read depth is calculated using the depth function of samtools with the -aa option If FADU is set to calculate fragment depth, all non-properly-paired reads are discarded by default and the read depth is adjusted to determine the fragment depth at all positions. The user can elect to keep all mapped read (as opposed to properly paired reads), including singletons and discordant reads, in which case all reads will be included in the fragment depth totals. To calculate the fragment depth from the samtools depth output, for each of the properly-paired reads, all coordinates in the insert region between the paired reads are incremented by one and coordinates where the reads overlap are decremented by one. If BAM data is identified as "stranded" or "reverse-stranded", each BAM file is split into a "(+)-stranded" and a "(-)-stranded" BAM file, based on the bitwise flag field in the input BAM file.

107 Each stranded BAM will have its read or fragment depth calculated separately. 
111 For each feature, all the coordinates that mapped solely to this feature are collected. The total depth of

112 this feature is calculated by summing the read or fragment depth for each coordinate collected in the

113 feature, and this total is divided by the average read or fragment length to derive a fragment count for

114 each feature. The feature ID and count statistic is written to a file. If multiple BAM files were used as

115 input, then the counts of each individual input will be written to a separate file.

\section{Implementation}

117 FADU was written entirely using the Python3 programming language. It relies heavily on the PySam

118 module (version 0.12.0.1 or later) to parse information from the BAM alignment files, to write

119 intermediate BAM files, and to perform basic samtools commands. The program supports

120 multiprocessing, and the user can specify the number of processes to be utilized. Each process will

121 handle a separate BAM input file if a list of files is provided. FADU was tested in the UNIX environment.

122 To minimize the amount of memory used, temporary files are written when possible to keep track of

123 read depth and the coordinates of properly paired reads. In addition, when read depth is converted into

124 fragment depth, only the bases with nonzero depth are read into memory. 


\section{$125 \quad 5$ Results}

\section{$126 \quad 5.1$ Gene detection performance of FADU, HTSeq, and featureCounts}

127 To assess how FADU compares to featureCounts and HTSeq in deriving counts, we used paired-end

128 sequencing data from three different sets of transcriptome data: (a) paired end reads from a standard

129 (i.e. not strand-specific) library constructed from Escherichia coli RNA, (b) paired end reads from a

130 standard library constructed from Ehrlichia chaffeensis RNA, and (c) paired-end reads from a strand-

131 specific library constructed from Wolbachia endosymbiont of Brugia malayi wBm RNA.

132 Of the 4,647 protein-coding genes detected in E. coli, counts for 51 genes could be obtained using FADU,

133 but not HTSeq union, the default HTSeq mode (Figure 2a). Because HTSeq union discards fragments

134 spanning multiple features, in the case when unstranded data is being used, HTSeq union is likely unable

135 to identify these genes because: (1) the gene is largely overlapping another feature either on the same

136 or opposite strand or (2) the gene is within an operon and smaller than the average library fragment

137 size. Because FADU calculates counts based on the depth at only positions unique to any given feature,

138 FADU can assign partial counts to multiple features per fragment, allowing for the increased

139 representation of smaller genes, as well as the unique portion, if any, of overlapping genes. Supporting

140 this, 42 of the 51 genes unable to be detected with HTSeq union are $\leq 300$ bp in size (Table 1). While

141 HTSeq union and featureCounts are largely similar, featureCounts handles ambiguous reads differently.

142 Given a fragment that maps to multiple features, featureCounts will assign the paired-end fragment to

143 the feature that maps to the majority of the individual paired-end reads (Liao, et al., 2014). When

144 comparing FADU with featureCounts. 21 genes were only detected using FADU, with 18 of these genes

145 being $\leq 300 \mathrm{bp}$ in size (Figure $\mathbf{2 b}$ ).

146 Similarly, FADU can derive counts for an additional five genes in E. chaffeensis compared to HTSeq union

147 or an additional two genes when compared to featureCounts. All genes detected only with FADU in $E$. 
148 chaffeensis were $\leq 300$ bp in length. With $w \mathrm{Bm}, 31$ additional genes were detected in FADU when

149 compared to HTSeq union, of which 10 are $\leq 300 \mathrm{bp}$, while 24 additional genes were detected when

150 compared to featureCounts, of which 7 are $\leq 300$ bp (Figure $2 \mathrm{ab}$ ). This indicates that despite

151 featureCounts being able to detect a greater number of genes than HTSeq union, FADU can derive

152 counts for genes that neither HTSeq or featureCounts can by default.

153 HTSeq has two additional modules to derive counts for transcriptome data that both attempt to assign

154 ambiguous reads. In the case that a fragment overlaps multiple features, HTSeq intersection-nonempty

155 takes the intersect of the features found at each non-empty position and if only one feature is returned,

156 a count is assigned to that feature. Similarly, HTSeq intersection-strict takes the intersect of the features

157 found at all positions of the fragment and again, if only one feature is returned, a count is assigned to

158 that feature (Anders, et al., 2015). While this allows for the assignment of more ambiguous fragments,

159 smaller genes are still under-represented. Additionally, because HTSeq intersection-strict also discards

160 fragments that partially map to intergenic regions, and because most prokaryotic organisms currently

161 have no UTR annotations, this will result in discarding reads at the 5'- and 3'-end of prokaryotic

162 transcripts. In all cases, for genes smaller than the library insert size, it becomes difficult to extract any

163 meaningful fragment counts.

164 When comparing FADU to HTSeq intersection-strict, FADU derives counts for an additional 182 genes.

165 HTSeq intersection-strict fails to obtain counts for $>100$ additional genes compared to HTSeq union

166 (Supplementary Figure 1ab), confirming the inability of HTSeq-intersection-strict to accurate assess

167 prokaryotic transcriptome data for instances in which the reference has limited UTR annotations.

168 Supporting this, HTSeq intersection-strict fails to detect an additional 60 genes in E. chaffeensis and 71

169 genes in wBm when compared to FADU. HTSeq intersection-nonempty performs similarly to HTSeq

170 union, failing to detect 48, 4, and 31 genes when compared to FADU in E. coli, E. chaffeensis, and wBm, 
171 respectively, indicating regardless of which module used, HTSeq is too conservative in assigning reads to

172 genes.

173 While featureCounts does not have any distinct modules, there are two options which help to assign

174 counts for ambiguous reads. The first is the -O option, in which cases where a fragment overlaps

175 multiple features, a single count is added to both. The second is specifying both the -O and the -

176 fragment options, in which case fragments that overlap $x$ features are given a count of $1 / x$. For the $E$.

177 chaffeensis, and wBm datasets, FADU obtains counts for the same number of genes as both

178 featureCounts overlap and featureCounts fractional-overlap (Supplementary Figure 2ab). However, in

179 the E. coli dataset, both modes of featureCounts have counts for nine additional genes compared to

180 FADU. Of these nine E. coli genes, eight are completely overlapped by another gene either on the same

181 or opposite strand. Because these genes have no unique positions with which FADU can use to

182 determine count values, FADU returns a fragment count of 0 for these genes. The last gene,

183 E2348C_0713, is 642 bp long with the first 104 bp being overlapped by another gene. At most,

184 featureCounts overlap gives E2348C_0713 a fragment count of 2, while featureCounts fractional-overlap

185 assigns a count of 1, indicating that only two fragments map to E2348C_0713 map within the first 104

186 bp. Because FADU calculates fragment counts using only unique positions of a gene, FADU assigns a

187 fragment count of 0 to E2348C_0713.

\subsection{Comparative analysis of FADU, HTSeq, and countFeatures in $w B m$}

Using the wBm dataset, we sought to determine the similarity of FADU compared to each of the

different modes of HTSeq and featureCounts. Fragment count values from the three HTSeq modules,

191 three featureCounts modes, and FADU were used for a clustering analysis. An unrooted dendrogram of

192 the different tools shows three distinct groups that cluster on how each of the different tools handle

193 fragments mapping to multiple features (Figure 3a). FADU, featureCounts overlap, and featureCounts

194 fractional-overlap, which are more liberal in assigning counts, form a cluster while HTSeq union, HTSeq 
intersection-nonunique, and featureCounts default, which are all more conservative, form another

196 cluster. HTSeq intersection-strict clusters with neither of the groups, due to it being the most stringent

197 in assigning fragment counts to features.

198 A heatmap showing counts from each of the eight tools shows HTSeq intersection-strict to have the 199 greatest number of genes with no assigned counts (Figure $\mathbf{3 b}$ ). Only genes with derived count values from at least one tool are shown. The cluster containing featureCounts default, HTSeq union, and HTSeq

201 intersection non-empty contain slightly less genes with no assigned count values while the cluster

202 containing FADU, featureCounts overlap and featureCounts fractional-overlap contain the least.

203 Although featureCounts overlap is able to assign count values to the same number of genes as FADU, it over-counts genes by assigning a full count value to all genes overlapped by a single fragment. In the case of a fragment overlapping a two gene operon, featureCounts overlap would assign a full count to both, despite there only being a single fragment. To diminish over-counting, featureCounts fractionaloverlap instead assigns a fractional count value based on the number of features a fragment overlaps.

208 While this alleviates the issue, featureCounts fractional-overlap implies that all features overlapped by a 209 fragment contribute equally to the fragment, which may not necessarily be true. The problem is particularly acute if the overlap is a relatively small fraction of the feature. FADU assigns count values

211 based on the percentage of the fragment that is overlapped, such that a higher partial read count is 212 assigned to the gene with the greater overlap. By doing so, FADU can assign higher counts from 213 ambiguous fragments to genes that the fragment most likely originated from, while still being able to 214 derive counts for smaller genes.

215 A principal component analysis of the counts show less discrete clusters compared to those seen in the 216 unrooted dendrogram (Figure 3c). While the counts from HTSeq union and HTSeq intersection-

217 nonempty are grouped together, no other two counts cluster closely with another. In the first principal 218 component, which accounts for $68.0 \%$ of the variation observed in the counts, the top 20 contributing 
genes are primarily represented by genes with lower counts in the three HTSeq modes relative to

220 featureCounts and FADU (Supplementary Figure 3a). Similarly, the top 20 contributing genes in the

221 second principal component, which accounts for $23.9 \%$ of the variation observed, separates the HTSeq-

222 derived counts from the featureCounts and FADU counts (Supplementary Figure 3b). In both principal

223 components, there are genes with lower counts in HTSeq intersection-strict relative to all other counts,

224 reflecting the conservative nature in which it assigns counts. Because of how featureCounts overlap

225 derives counts, it will always have greater than or equal to the highest number of counts relative to all

226 other algorithms tested.

\section{Discussion}

228 During transcript quantification for RNA-Seq analyses, the handling of fragments that overlap multiple

229 features must be addressed. This may not be as much of an issue in many eukaryotes, where genes are

230 larger and spaced further apart. But in prokaryotes, the closer proximity of genes coupled with the

231 presence of operons leads to a large number of fragments being classified as ambiguous. Tools such as

232 HTSeq and featureCounts have different modules and/or options to handle these ambiguous fragments,

233 but smaller genes, especially those in operons, become either under- or over-represented depending on

234 the tool. In this study, we present FADU, a novel tool for transcript quantification in RNA-Seq analyses

235 that addresses these issues.

236 While it can be easy to think of all Illumina data as being equal, our analysis suggests that small genes

237 near or below the insert size of the library are specifically being lost. This bears more scrutiny and

238 consideration in prokaryotic transcriptomic sequencing projects, since the insert size of the library varies

239 between samples and is not frequently reported. Our results suggest that these small genes could be

240 differentially reported, in a purely artefactual way, during feature counting and impacts downstream

241 analyses, like differential expression, clustering, and PCA-type analyses. 
242 Importantly, FADU is not a counting algorithm and as such does not report counts as other algorithms

243 have over the past several years. As such, it does not return integers, instead returning fraction-based

244 rational numbers. As such the output of FADU cannot be used in downstream tools that require integer

245 counts, such as some differential expression analysis tools. It can, however, be used with success in

246 edgeR and in calculating TPMs and z-scores. There may, however, be a new need for further

247 development of statistical analysis tools that do not require integer-based data.

248 Compared to the default HTSeq and featureCounts modes, which largely discard ambiguous reads, FADU

249 assigns partial read counts based on the percentage of the fragment that is within the unique positions

250 of gene. By doing this, FADU is able to assign partial counts to features that are missed by both HTSeq

251 and featureCounts by default. While HTSeq and featureCounts have options that allow for the

252 assignment of reads to these features, we find that both the overlap and fractional-overlap options

253 overstate their abundance, especially in the case of completely overlapped genes. FADU weighs the

254 percentage of each fragment covered by a feature so in the case that a fragment does overlap multiple

255 features, instead of assigning equal counts to both features, partial read counts are assigned based on

256 the percentage of the fragment covered by the feature. Due to its ability to derive counts for otherwise

257 unrepresented genes without overstating their abundance, we believe FADU to be an improved tool for

258 quantifying transcripts in prokaryotic systems for RNA-Seq analyses. 
bioRxiv preprint doi: https://doi.org/10.1101/337600; this version posted June 3, 2018. The copyright holder for this preprint (which was not certified by peer review) is the author/funder, who has granted bioRxiv a license to display the preprint in perpetuity. It is made available under aCC-BY 4.0 International license.

\section{Acknowledgements}

260 This project was funded in part by federal funds from the National Institute of Allergy and Infectious

261 Diseases, National Institutes of Health, Department of Health and Human Services under grant number

262 U19 Al110820. 


\section{References}

264 Anders, S. and Huber, W. Differential expression analysis for sequence count data. Genome Biol

$2652010 ; 11(10): R 106$.

266 Anders, S., Pyl, P.T. and Huber, W. HTSeq--a Python framework to work with high-throughput

267 sequencing data. Bioinformatics 2015;31(2):166-169.

268 Dobin, A., et al. STAR: ultrafast universal RNA-seq aligner. Bioinformatics 2013;29(1):15-21.

269 Kim, D., Langmead, B. and Salzberg, S.L. HISAT: a fast spliced aligner with low memory requirements.

$270 \quad$ Nat Methods 2015;12(4):357-360.

271 Langmead, B., et al. Ultrafast and memory-efficient alignment of short DNA sequences to the human

272 genome. Genome Biol 2009;10(3):R25.

273 Le, S., Josse, J. and Husson, F. FactoMineR: An R Package for Multivariate Analysis. Journal of Statistical

274 Software 2008;25(1):1-18.

275 Lewis, M. Allostery and the lac Operon. J Mol Biol 2013;425(13):2309-2316.

$276 \mathrm{Li}, \mathrm{H}$. and Durbin, R. Fast and accurate short read alignment with Burrows-Wheeler transform.

277 Bioinformatics 2009;25(14):1754-1760.

278 Liao, Y., Smyth, G.K. and Shi, W. featureCounts: an efficient general purpose program for assigning

279 sequence reads to genomic features. Bioinformatics 2014;30(7):923-930.

280 Paradis, E., Claude, J. and Strimmer, K. APE: Analyses of Phylogenetics and Evolution in R language.

281 Bioinformatics 2004;20(2):289-290.

282 Popescu, A.A., Huber, K.T. and Paradis, E. ape 3.0: New tools for distance-based phylogenetics and

283 evolutionary analysis in R. Bioinformatics 2012;28(11):1536-1537.

284 Robinson, M.D., McCarthy, D.J. and Smyth, G.K. edgeR: a Bioconductor package for differential

285 expression analysis of digital gene expression data. Bioinformatics 2010;26(1):139-140. 
bioRxiv preprint doi: https://doi.org/10.1101/337600; this version posted June 3,2018. The copyright holder for this preprint (which was not certified by peer review) is the author/funder, who has granted bioRxiv a license to display the preprint in perpetuity. It is made available under aCC-BY 4.0 International license.

286 Suzuki, R. and Shimodaira, H. Pvclust: an R package for assessing the uncertainty in hierarchical

287 clustering. Bioinformatics 2006;22(12):1540-1542.

288 


\section{Table 1. Key Properties of Data Examined}

\begin{tabular}{llll}
\hline Species & E. coli & E. chaffeensis & Bm \\
\hline Strand-Specificity & no & no & reverse \\
Number of Sequenced Paired-End Reads & $215,149,159$ & $46,817,709$ & $75,945,674$ \\
Number of Mapped Paired-End Reads & $184,454,369(85.7 \%)$ & $3,132,709(6.7 \%)$ & $351,928(0.5 \%)$ \\
Genes & 4,647 & 1,002 & 1,006 \\
$\begin{array}{l}\text { Genes Detected in FADU but not HTSeq } \\
\text { or featureCounts }\end{array}$ & 51 & 5 & 31 \\
\hline
\end{tabular}

290 


\section{Figure Legends}

\section{Figure 1: Comparison of TPM values derived from FADU to HTSeq union and 293 featureCounts default}

294 For three different sets of RNA-Seq paired-end data from E. coli, E. chaffeensis, and wBm, the $\log _{2}$ TPM

295 values for genes quantitated using FADU were plotted against the $\log _{2}$ TPM values for genes quantitated

296 with (A) HTSeq union and (B) featureCounts default. Each point is representative of a single gene, with

297 points in blue being representative of genes $\leq 300 \mathrm{bp}$ in length. Genes with similar count values are

298 expected to lie close to the identity line ( $x=y$; red). Genes whose expression values are more elevated in

299 FADU lie above the identity line while genes whose expression values are elevated in HTSeq of

300 featureCounts lie below the identity line. Genes able to be quantified in FADU but not in HTSeq union or

301 featureCounts default lie on the $y$-axis. These genes include very highly transcribed genes suggesting

that they are missed by all the tools except FADU, and not that they are poorly transcribed, small genes.

Figure 2: Clustering patterns of the different count values in wBm derived with HTSeq modules, featureCounts modes, and FADU

(A) An unrooted dendrogram with 1000 bootstraps was generated using the $\log _{2}$ count values from

wBm calculated using HTSeq, featureCounts, and FADU. The dendrogram reveals three distinct clusters

values for all $w \mathrm{Bm}$ genes with count values derived from at least one of the tools was used to generate a

311 the vertical axis. All cells in grey describe genes with no count value in its corresponding tool. Bootstrap

312 values for both the unrooted and squared dendrograms are located next to their corresponding nodes.

313 (C) A principal component analysis for all $w \mathrm{Bm}$ count values derived from each of the tools was done.

314 Each color corresponds to either FADU, HTSeq, or featureCounts, while each shape represents the

315 specific mode of the tool used. 


\section{Supplementary Figure 1: Comparison of TPM values derived from FADU to 317 HTSeq intersection-nonempty and intersection-strict}

318 For each of the three different sets of RNA-Seq paired-end data from E. coli, E. chaffeensis, and wBm, the $\log _{2}$ TPM values for genes quantified using FADU were plotted against the $\log _{2}$ TPM values for genes quantified with two of the non-default HTSeq modules: (a) HTSeq intersection-nonempty and (b) HTSeq

321 intersection-strict. Each point is representative of a single gene, with points in blue being representative

322 of genes $\leq 300 \mathrm{bp}$ in length. Genes with similar count values are expected to lie close to the identity line

323 ( $\mathrm{x}=\mathrm{y} ; \mathrm{red}$ ). Genes whose expression values are more elevated in FADU lie above the identity line while

324 genes whose expression values are elevated in the HTSeq counterpart lie below the identity line. Genes

325 able to be quantified with FADU but not in HTSeq lie on the $y$-axis.

\section{Supplementary Figure 2: Comparison of TPM values derived from FADU to} 327 featureCounts overlap and fractional-overlap

328 For each of the three different sets of RNA-Seq paired-end data from E. coli, E. chaffeensis, and wBm,

329 the $\log _{2}$ TPM values for genes quantitated using FADU were plotted against the $\log _{2}$ TPM values for

330 genes quantitated with two different featureCounts runs. (a) The first set of plots are run with the

331 featureCounts option overlap, in which multiple genes overlapped by the same fragment are both

332 assigned full counts. (b) The second set of plots are run with the featureCounts option overlap and

333 fractional, in which multiple genes overlapped by the same fragment are assigned fractional counts

334 depending on the number of features overlapped by the fragment. Each point is representative of a

335 single gene, with points in blue being representative of genes $\leq 300 \mathrm{bp}$ in length. Genes with similar

336 count values are expected to lie close to the identity line ( $x=y$; red). Genes whose expression values are

337 more elevated in FADU lie above the identity line while genes whose expression values are elevated in

338 its featureCounts counterpart lie below the identity line. Genes able to be quantified in featureCounts

339 overlap or fractional-overlap but not FADU lie on the $x$-axis. 


\section{Supplementary Figure 3: Clustering of the twenty top contributing wBm genes in} 341 the first and second principal components

342 Two heatmaps were generated to visualize the top contributing in (a) the first and (b) the second

343 principal components analysis of the variation in counts for wBm genes derived using HTSeq,

344 featureCounts, and FADU. For each of the two principal components, the top twenty contributing genes

345 to the variation observed are shown. The horizontal axis of the heatmap describes the tool used while

346 each of the genes are indicated on the vertical axis. The $\log _{2}$ count values are shown in each of the

347 corresponding cells. 
E. coli

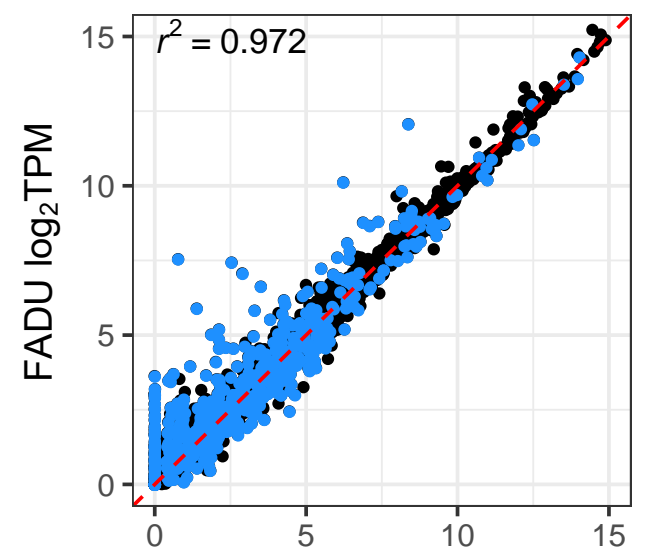

B
E. chaffeensis

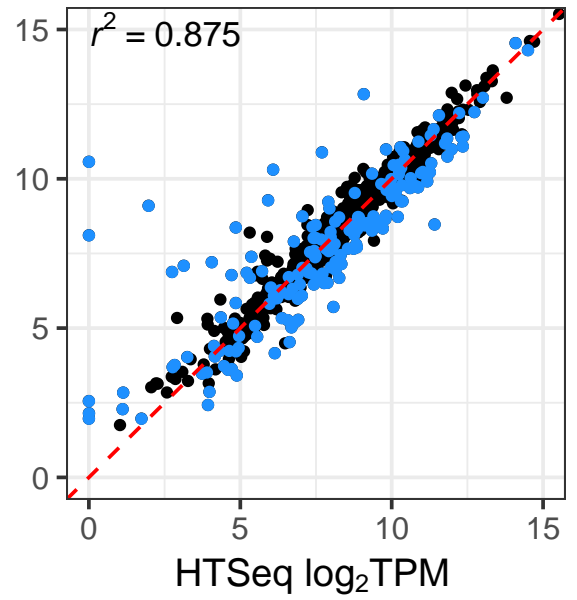

E. chaffeensis

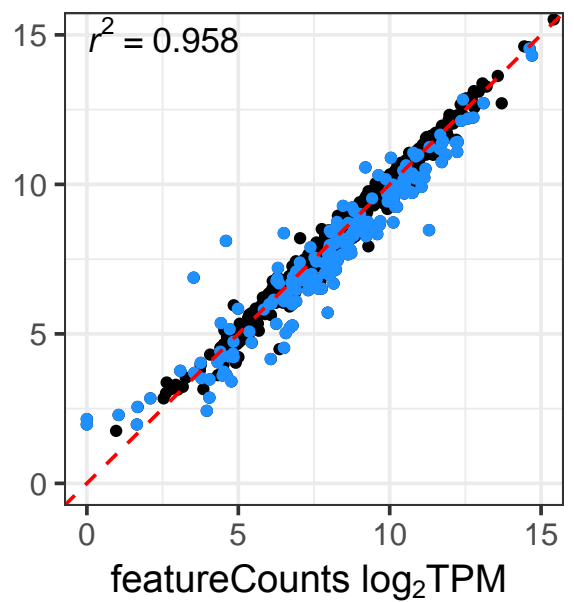

wBm

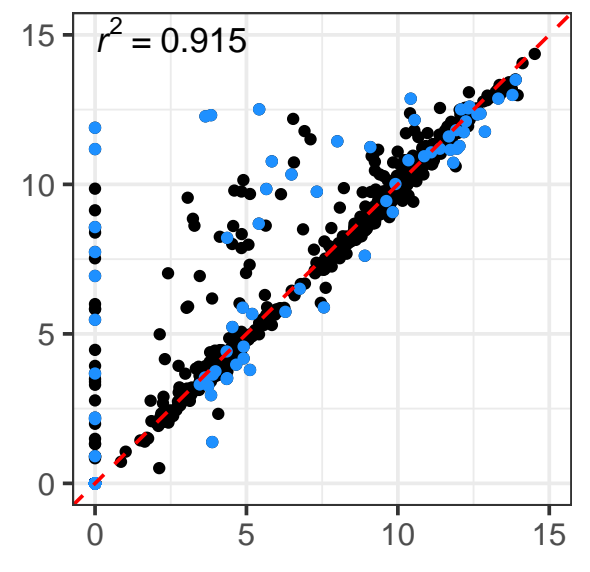

wBm

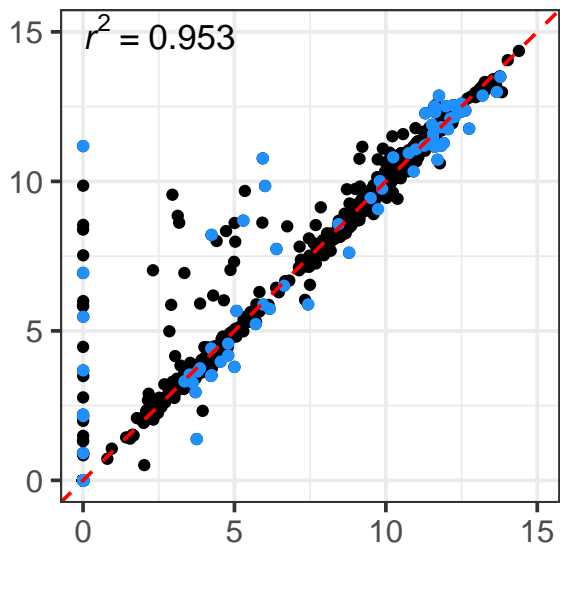



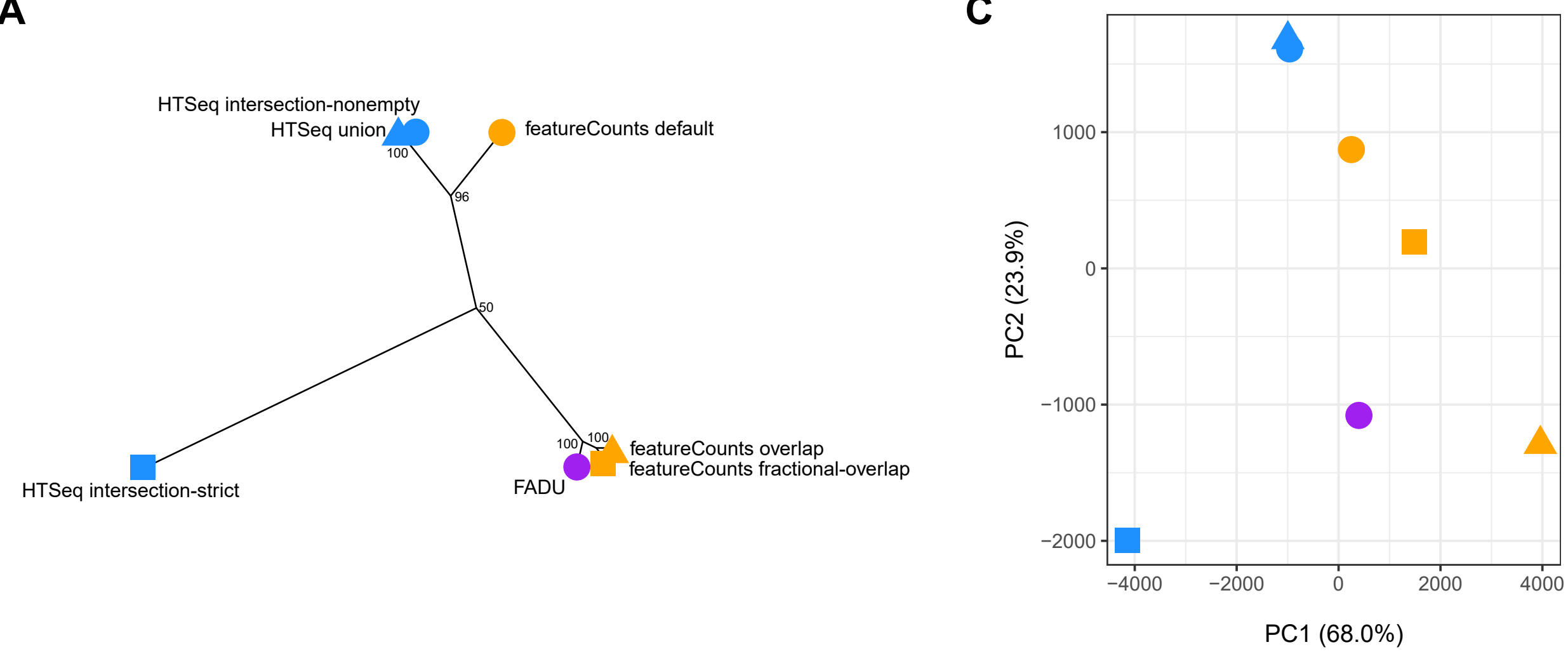

featureCounts default

featureCounts fractional-overlap

featureCounts overlap

HTSeq intersection-nonempty

HTSeq intersection-strict

HTSeq union

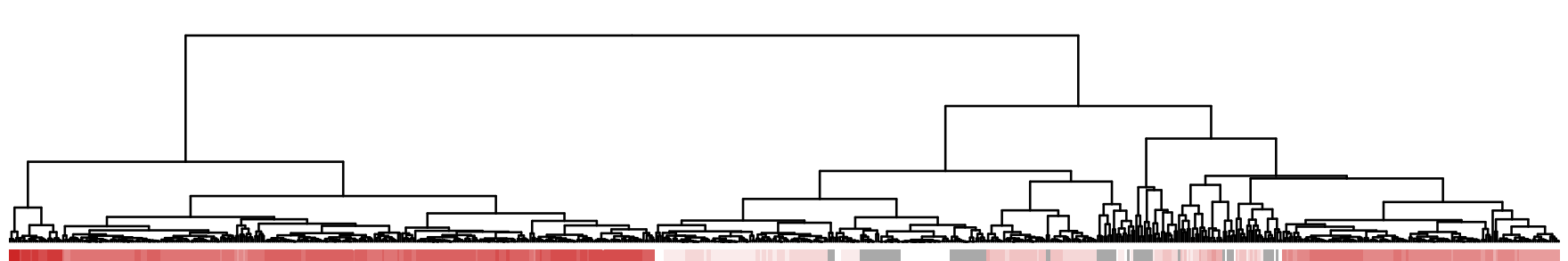

\section{HTSeq intersection-strict}

FADU

featureCounts overlap featureCounts fractional-overlap featureCounts default HTSeq intersection-nonempty HTSeq union
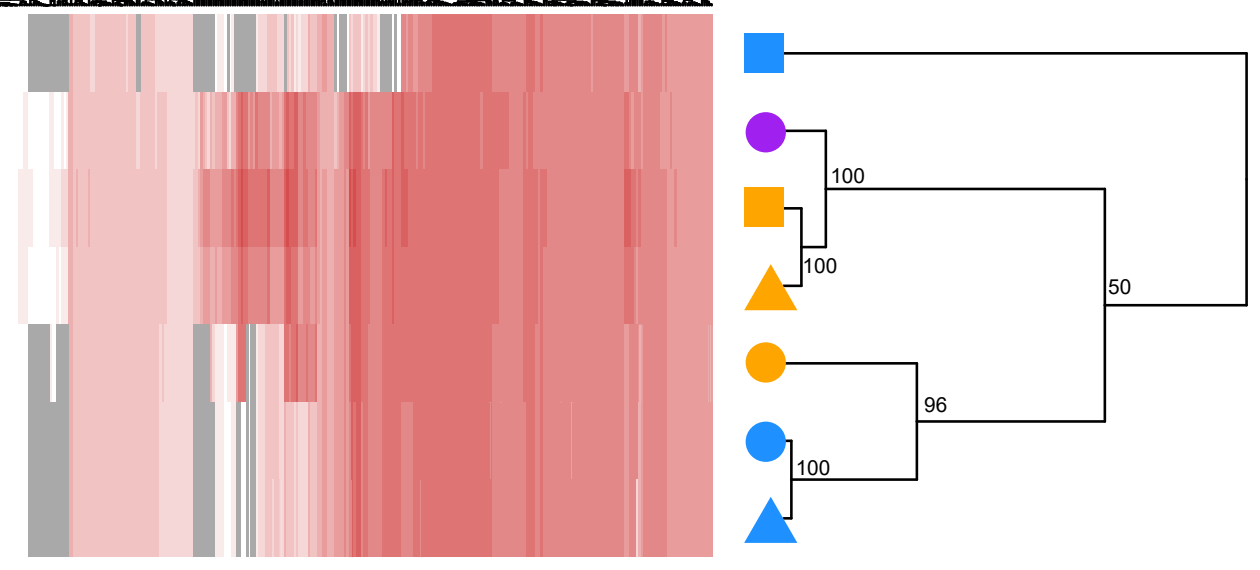